\title{
QoS Aware Optical Virtual Private Network (OVPN) Analytical Control Plane Mechanism
}

\author{
Santos Kumar Das and Sarat Kumar Patra, Senior Member, IEEE
}

\begin{abstract}
Due to the demand of clients, the service provider network needs to provide functionalities such as dynamic OVPN connection provisioning, de-provisioning and rerouting in order to provide guaranteed Quality of service (QoS). In corporate with that, we present a centralized OVPN control mechanism over DWDM/GMPLS network based on the existing OVPN traffic. It is important to understand the process that provide the QoS information to the centralized Traffic control manager and use this information efficiently to compute feasible OVPN connection for the prospective of client OVPN connection requirements.
\end{abstract}

Index Terms - Bandwidth, delay, traffic control manager, OVPN provisioning, QoS, link failure, OVPN connection

\section{INTRODUCTION}

In this work we consider the problem of providing guaranteed service, provisioning and de-provisioning of OVPN connection (OVPNC) of the Electro-Optical Network [1]. This Electro-optical Network supports multimedia applications having various QoS requirements of the OVPN clients. Guaranteed Quality of Service (QoS) traffic requires a good traffic engineering control model, which can be applied at any router. This traffic engineering control model can be centralized or distributed, where the distributed traffic engineering control model is more scalable than centralized one. In our proposed model, we consider the case of centralized model. This model considers different QoS constraints, like available bandwidth and end-to-end delay for the guaranteed services. Such application requires the network to provide a wide range of QoS guarantees. Whereas the guaranteed bandwidth must be large enough to accommodate motion video of acceptable resolution, end-to-end delay must be small enough for interactive communication. In order to avoid breaks in continuity of audio and video playback, delay jitter and loss must be sufficiently small.

In this paper, we employ Optical Virtual Private Network (OVPN). This is also known as layer one VPN (L1VPN) [2] Optical Virtual Private Network (OVPN) provides service to the customer in the form of optical connection, which also expected to be one of the major applications in future optical networks. OVPN can be a favorable approach for realizing the next generation virtual private network (VPN) services [3] [4] by providing a guaranteed QoS [5]. In our work, the QoS requirements of the OVPN client have been considered in

Manuscript received April 4, 2012; revised May 20, 2012. This work was supported in part by the National Institute of Technology, Rourkela, India

The authors are with the National Institute of Technology, Rourkela, India (e-mail: dassk@nitrkl.ac.in). terms of bandwidth and delay. We specify our network model based on all those QoS parameters by providing an end-to-end delay bound [6], [7] model based on the source-destination OVPN traffic characteristics. In this paper, we have mentioned bandwidth and end-to-end delay model, with a simple method for the provisioning of OVPN connection for a set of applications. The main objectives of this paper is to when and how to provision and de-provision an OVPN connection for the incoming traffics at the access router. We solve these problems by formulating a mathematical admission control model and a traffic aggregation model at the provider edge router (PER), which can solve the problem of traffic optimization at the access router.

In addition that, we have Traffic Engineering model based on the idea of differentiated services (DiffServ) [8] to maintain the quality of services of every incoming traffic. This DiffServ architecture achieves scalability by aggregating traffic classification state, which is conveyed by means of IP-layer. This architecture achieves scalability by implementing complex classification and conditioning functions at network optical routers (ORs), and by applying per-hop behaviours to aggregates of traffic. Per-hop behaviours are defined to permit a reasonably granular means of allocating buffer and bandwidth resources at every OR among competing traffic streams. Per-application flow or per-client forwarding state need not be maintained within the core of the network.

In this research work we will consider a GMPLS capable hybrid network with general purpose optical routers (OR), which is the combination of IP and WDM network. The ORs supports QoS guarantees and may be used as access or gateway routers for optical switching equipment leading to the core transport network. Based on the global monitoring and control information of the Traffic Control Manager (TCM), it is proposed an OVPNC control mechanism for the provisioning and de-provisioning of OVPNCs. The work will focus on GMPLS techniques, which is defined as the IP based control of optical resources, where ORs are expected to deliver advanced services at higher throughput while handling large amounts of label switching path (LSP) creation/deletion, traffic engineering link information exchange, and link state advertisements. The novelty of this work lies in the fact that it combines provisioning and de-provisioning of OVPNC for the hybrid network under a common framework, using the hybrid information obtained by monitoring the ORs in the network. This technique will be useful to service provider networks, where both IP and WDM network domains are owned or leased by the same operator. Using these techniques the service provider can optimally utilize his network of ORs and optical gear for better 
utilization of resources. In the next section, the network model is introduced.

There are lots of similar works has been published from which some of them we mentioned. RSVP [9] defines a purely flow based protocol to ensure about the individual flow requirements. The differentiated services architecture [8] works with aggregated flows based upon the notion of per hop behaviors. It takes static decision for the re-routing of specific flows. Another work Bandwidth broker [10], which is very close to our work. In all the above works the implication of end-to-end QoS support in Electro-Optical domain are not considered.

In next section a network model is introduced. In section 3, the problem formulation based on bandwidth model and delays model are described. In section 4, The OVPNC protection, provisioning and de-provisioning methods are presented. In section 5, analytical results and discussion are mentioned. Finally in section 6, some conclusions are drawn.

\section{OVPN SYSTEM MODEL}

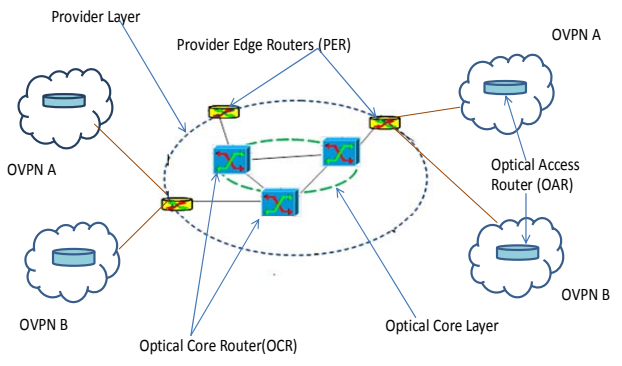

Fig. 1. OVPN system model

The model shown in Fig.1 shows the network topology consisting of two layers, the provider layer shown as the outermost layer and the Optical core layer which is the innermost Optical network layer. The provider layer is connected to the OVPN clients. This is an abstraction of the combined IP-optical network which allows us to focus on that portion of the network where our innovation applies, i.e. the combined IP-optical network. The optical layer provides point-to-point connectivity between routers in the form of fixed bandwidth circuits, which is termed as OVPNCs. The collection of OVPNCs therefore defines the topology of the virtual network interconnecting electronics/IP Routers. In IP layer the IP routers are responsible for all the non-local management functions such as management of optical resources, configuration and capacity management, addressing, routing, topology discovery, traffic engineering, and restoration etc. The IP router communicates with the TCM (Traffic Control Manager) of service provider network and provides the information about the status of the optical layer.

Ideally the service provider layer will include elements of the access network such as the PON (Passive Optical Network) related elements and other devices / equipment located at the premises / home. However for this invention such details are not necessary. We assume that the provider has access to optical core routers (OCRs) and also optical components in the core optical network. Such an assumption is reasonable, given the fact that the prices of optical switching equipment have fallen by orders of magnitude till the point that they are being used in the premises of large corporations in order to interconnect buildings etc. Thus it is reasonable to assume, as we have done, that the service provider has information about the ORs and the optical equipment within its domain of control. We assume all the routers are same type and known as optical routers (ORs).

The provider layer controls all the traffic corresponding to both electronic and optical layers. All the routers shown in the figure are controlled by the service provider (SP). The SP maintains a traffic matrix in a Traffic Control Manager (TCM) for all the connected ORs within its domain of control.

The Traffic Control Manager (TCM) maintains the network state information (bandwidth and delay matrices) for all the ORs in the network, belonging to all domains, electronics, optical and electro-optical domains. In the following sections we outline our algorithms that carry out the computations necessary for the decisions that lead to provisioning/de-provisioning of OVPNCs.

\section{PROBLEM FORMULATION}

We consider a virtual topology model shown in Fig. 2. In this model, a number of flows for different applications are multiplexed at the source OR $s$ to destination OR $d$, for an OVPN client requirement. This formulation is for the provisioning and de-provisioning of OVPNC based on the OVPN client requirements and existing traffic. The problem formulations are based on different QoS parameters such as, bandwidth and delay bound, which are explained in following sections.

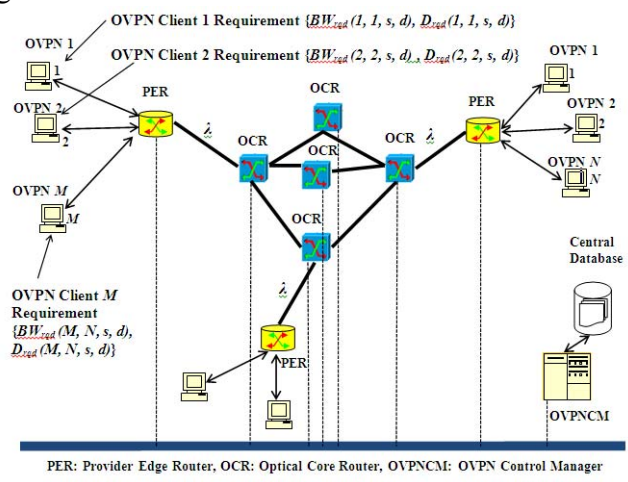

Fig. 2. Physical topology

\section{A. Bandwidth Model}

Assumption every OVPN client end point is attached to at most one PER. Suppose an OVPN connection for client $m$ and $n$ with OVPNC from source $s$ to destination $d$ has bandwidth requirement $B_{r q d}(m, n, s, d)$. The aggregated bandwidth for the all OVPN connection request from the OVPN clients can be computed as follows:

$$
B W_{A g g r}(m, n, s, d)=\sum_{m, n} B_{r q d}(m, n, s, d)
$$

A free available/computed bandwidth matrix, $B(m, n, s, d)$ has been considered for every PER. If $D S(i, j)$ is the dispersion of the fiber and $L(i, j)$ is the length of fiber link pair $(i, j)$, then the bandwidth matrix for this link can be represented [11] mathematically, 


$$
B(i, j)=\frac{\delta}{D S(i, j) \times \sqrt{L(i, j)}}
$$

where, $\delta$ represents the pulse broadening factor should typically be less than $10 \%$ of a bit's time slot for which the polarization mode dispersion (PMD) can be tolerated [12] and $D S(i, j)=L(i, j)=\infty$, when there is no link from $i$ to $j$.

The bandwidth matrix for an OVPN connection will be:

$$
B(m, n, s, d)=\operatorname{Min}\{B(i, j)\}, \forall(i, j) \in p
$$

where, $p$ is the computed OVPN connection for a source and destination pair $(s, d)$, which is a group of links.

\section{B. Delay Bound Model}

Here we derive how to express the delay requirement of the clients in terms of maximum acceptable delay as well as computed delay of the OVPNC in terms of maximum end-t-end delay bound. In Figure 2, suppose the delay requirement of flow for $(m, n)$ client pair from source $s$ and destination $d$ is $D(m, n, s, d)$. The maximum acceptable delay between $i$ and $j$ is as follows:

$$
\begin{aligned}
& D_{\max }^{\text {accept }}(m, n, s, d)=\operatorname{Minimum}\left\{D_{r q d}(m, n, s, d)\right\}, \\
& \forall(m, n), m=1,2,3, \ldots, M \text { and } n=1,2, \ldots, N
\end{aligned}
$$

where, $M$ and $N$ are the total number clients attached to source $s$ and $d$ respectively. If $D(i, j)$ is the link wavelength dependent local delay, then the end-to-end delay bound is the sum of all maximum local delays suffered by a connection at all the routers along with the path $(p)$ and given as:

$$
D_{\max }^{\text {bound }}(m, n, s, d)=\sum_{(i, j \in \in p} D(i, j)
$$

where, according to [13],

$$
D(i, j)=a \lambda_{i, k}^{2}+b+c \lambda_{i, k}^{-2}
$$

where, $a, b$ and $c$ are fiber material dependant constants, $\lambda_{i, k}$ is the wavelength at $i^{\text {th }}$ node/router and its $k^{\text {th }}$ connections.

\section{Link Failure Model}

Assume there is only one connection from source $s$ and $d$. If there is a link failure for this connection, then the link length will be changed to $L \quad(i, j)=\infty$. The bandwidth metrics $B(m, n, s, d)$ can be represented as from equation 3 as follows:

$$
B(m, n, s, d)=0, \forall(m, n)
$$

\section{OvPn PROTECTION AND PROVISIONING MECHANISM}

The PER aggregates the bandwidth requirements of the OVPN connection requests for source-destination edge pairs. This aggregated bandwidth will be compared with the computed/available bandwidth of the OVPN connection, which shows the available bandwidth of each existing OVPN connection of their corresponding. The comparison takes decision, whether to provision or de-provision an OVPN connection for the requested services.

\section{A. Provisioning of OVPNC before Link Failure}

Provisioning of OVPN connections should occur whenever the following conditions are satisfied.

$$
\begin{aligned}
& D_{\max }^{\text {accept }}(m, n, s, d) \geq D_{\max }^{\text {bound }}(m, n, s, d) \\
& B_{A g g r}(m, n, s, d)<B(m, n, s, d)
\end{aligned}
$$

The bandwidth constraint $B(m, n, s, d)$ changes based on above equations as follows:

$$
B(m, n, s, d)=B(m, n, s, d)-B_{A g g r}(m, n, s, d)
$$

\section{B. Protection and Provisioning of OVPN connection during Link Failure}

A threshold value has been considered at every PER for the better use of OVPN connection. Whenever the aggregated bandwidth becomes less than the threshold value, the corresponding OVPN connection should be dropped from the configuration.

The de-provisioning of an OVPN connection should occur whenever the following condition is satisfied:

$$
\begin{gathered}
B_{A g g r}(m, n, s, d)<<B(m, n, s, d) \\
B(m, n, s, d)=0
\end{gathered}
$$

Equation 12 says that the available bandwidth will be zero, when there is link failure. Whenever the aggregated bandwidth at the PER is very low as compared with the threshold value and if there is no more OVPN request for the existing OVPN connection, the existing OVPNC will be treated as under-utilized and should be dropped or de-provisioned.

\section{ANALYTICAL RESUltS AND DisCUSSION}

We have taken this different scenario for the OVPN connection protection mechanism as follows.

\section{A. Scenario 1: Client Requirement doesn't Map with the Available Resources}

We assume two OVPN clients for the source and one OVPN client at the destination node.

In Fig. 3, the calculated aggregate bandwidth $\left(\mathrm{B}_{\mathrm{Aggr}}\right)$ at edge router 1 (ER1) is $25 \mathrm{GHz}$ (i.e., $10+15)$, available or computed bandwidth $\mathrm{B}(\lambda 1)$ based on existing OVPN traffic on $\lambda 1$ is $10 \mathrm{GHz}$ (i.e., $\mathrm{B}(\lambda 1)=30-20), \mathrm{B}(\lambda 2)$ on $\lambda 2$ is $30 \mathrm{GHz}$ (i.e., 50-20), accepted delay bound $\mathrm{D}^{\text {accpt }}$ is minimum of (4 $\mu \mathrm{s}$, $5 \mu$ s) i.e. $4 \mu \mathrm{s}$, available or computed delay Bound $\mathrm{D}^{\text {bound }}$ is $2 \mu \mathrm{s}$.

Comparing with $\lambda 1$ : In this case the $B_{A g g r}>B(\lambda 1)$ and $D^{\text {accept }}>\mathrm{D}^{\text {bound }}$, which says that there is no satisfaction of bandwidth but satisfaction of delay requirement only.

Comparing with $\lambda 2$ : In this case the $B_{\text {Aggr }}<B(\lambda 2)$ and 
$D^{\text {accept }}>D^{\text {bound }}$, which says that there is satisfaction of both bandwidth and delay requirement. So $\lambda 2$ is suitable for the client traffic requirements, therefore ER1 aggregate the OVPNC request on $\lambda 2$, which means $\lambda 2$ will be assigned to the clients.

\section{B. Scenario 2: Available Bandwidth doesn't Map with Required Traffic}

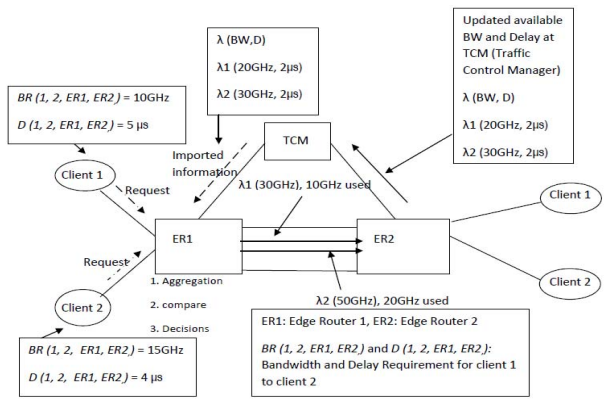

Fig. 3. Scenario 1

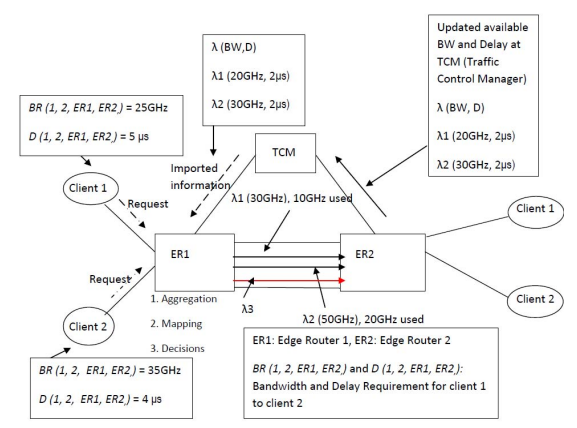

Fig. 4. Scenario 2

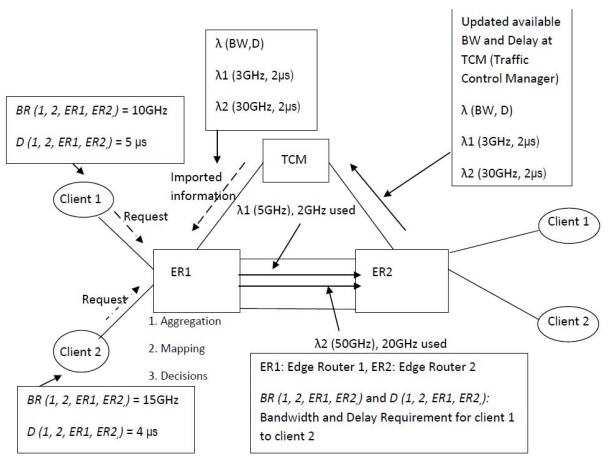

Fig. 5. Scenario 3

In Fig. 4 , the calculated $B_{A g g r}$ at ER1 is $60 \mathrm{GHz}$ (i.e., 25+35), $B(\lambda 1)$ is $10 \mathrm{GHz}$ (i.e., $30-20), B(\lambda 2)$ is $30 \mathrm{GHz}$ (i.e., $50-20$ ), $D^{\text {accep }}$ is minimum of $(4 \mu \mathrm{s}, 5 \mu \mathrm{s})$ i.e., $4 \mu \mathrm{s}, D^{\text {bound }}$ is $2 \mu \mathrm{s}$.

Comparing with $\lambda 1$ : In this case the $B_{A g g r}>B(\lambda 1)$ and $D^{\text {accept }}>D^{\text {bound }}$, which says that there is no satisfaction of bandwidth but satisfaction of delay requirement only.

Comparing with 22 : In this case the $B_{\text {Aggr }}>B(\lambda 2)$ and $D^{\text {accept }}>D^{\text {bound }}$, which says that there is no satisfaction of bandwidth and of delay requirement. In overall, there is no $\lambda$ is suitable for the client QoS requirement, therefore ER1 adds or provision a new connection with $\lambda 3$ for the aggregated OVPNC request.

\section{Scenario 3: Very low Traffic at a Particular Client}

This happens either during link failure or when required traffic flow is very low. In Fig. 5, Assume for Client 1 and Client 2, the required of bandwidths are $1 \mathrm{GHz}$ and $1 \mathrm{GHz}$ respectively, then $B_{\text {Aggr }}$ is $2 \mathrm{GHz}$ (i.e., $\left.1+1\right)$. In this scenario
$B_{\text {aggr }}<<B(\lambda 2)$ and $B_{\text {Aggr }}<B(\lambda 1)$ and hence the connection with $\lambda 1$ will be dropped or de-provisioned and switched to connection with $\lambda 2$.

\section{CONCLUSION}

We have proposed a mechanism that determines the time, when to add or drop an OVPNC for the connected clients to the provider DWDM/GMPLS network. What makes the mechanism unique is the fact that it performs traffic engineering on hybrid network, feeding rate allocation information to a traffic control manager, which performs a global optimization based upon the criteria, namely aggregated OVPN QoS requirements. The outcome of the global optimization is a new allocation rate and decision criteria to provision or de-provision of OVPNCs. Techniques adopted by IETF protocols do not perform global optimization. For example, RSVP is a purely flow based protocol and does not attempt to optimize resource consumption. Its major objective is to ensure that individual flow specifications are met. The differentiated services architecture does perform aggregation of flows based upon the notion of per hop behaviors; however this is a hard-wired approach. PHBs are defined ahead of time and flows are aggregated on this basis. Dynamic decisions regarding re-routing of specific flows cannot be taken. Furthermore, the scheme does not permit global optimization based on a flow matrix. Bandwidth broker is a third proposal, and perhaps comes closest to our scheme. However bandwidth brokers are purely based on the IP level and do not make use of information provided within the optical core of the network.

\section{ACKNOWLEDGMENT}

This research work is supported by National Institute of Technology, Rourkela, India. This support is gratefully acknowledged.

\section{REFERENCES}

[1] D. Khoe and H. V. D Boom, "Trends in Electro-Optical Communication System, Perspectives on Radio Astronomy: Technologies for Large Antenna Arrays," in Proceedings of the Conference held at the ASTRON Institute in Dwingeloo on 12-14 April 1999, pp. 285-293, ISBN: 90-805434-2-X , 2000.

[2] T. Takeda, "Framework and Requirements for Layer 1 Virtual Private Networks," IETF RFC 4847, April 2007.

[3] Z. Zhang, "An Overview of Virtual Private Network (VPN): IP VPN and Optical VPN," Photonic Network Communications, vol. 7, no. 3, pp. $213-225,2004$

[4] H. O. Brahim, "Generalized Provider-provisioned Port-based VPNs using BGP and GMPLS Toolkit," IETF Internet Draft, March 2003.

[5] H. Beyranvand and J. A. Salehi, "Multiservice Provisioning and Quality of Service Guarantee in WDM Optical Code Switched GMPLS Core Networks," journal of Lightwave Technology, vol. 27, no. 12, pp. 1754-1762, 2009.

[6] L. Georgiadis, R. Guerin, V. Peris, and R. Rajan, "Efficient Support of Delay and Rate Guarantees in an Internet," SIGCOMM'96, vol. 26, no. 4, pp. 106-116, ISBN:0-89791-790-1, 1996.

[7] J. Glasmann, M. Czermin, and A. Riedl, "Estimation of Token Bucket Parameters for Videoconferencing Systems in Corporate Networks," $8 T H$ International conference on computer software, Telecommunication and Computer Networks, (SoftCOM 2000), pp. $10-14,2000$.

[8] S. Blake, D. Black, M. Carlson, E. Davies, Z. Wang, and W. Weiss, "An Architecture for Differentiated Services," RFC 2475, December 1998. 
[9] L. Zhang, S. Deering, D. Estrin, S. Shenker, and D. Zappala, "RSVP: A New Resource Reservation Protocol," Communications Magazine, IEEE, vol. 14, no. 5, pp. 116-127, 2002.

[10] Z. Zhang, Z. Duan, L. Gao, and Y. T. Hou, "Decoupling QoS Control from Core Routers: A Novel Bandwidth Broker Architecture for Scalable Support of Guaranteed Services," ACM SIGCOMM 2000, pp. $71-83,2000$.

[11] Y. Huang, J. P. Heritage, and B. Mukherjee, "Connection Provisioning with Transmission Impairment Consideration in Optical WDM Networks With High-Speed Channels," Journal of Lightwave technology, vol. 23, no. 3, pp. 982-993, 2005.

[12] J. Strand, A. L. Chiu, and R. Tkach, "Issues for routing in the optical layer," Communications Magazine, IEEE, vol. 39, no. 2, pp. 81-87, 2001.

[13] Y. Miyajima, M. Ohnishi, and Y. Negishi, "Chromatic dispersion measurement over a $120 \mathrm{~km}$ dispersion-shifted single-mode fiber in the $1.5 \mu \mathrm{m}$ wavelength region," NTT Electrical Communications Laboratories, Tokai, Japan, Electronics Letters, 22, vol. 22, no. 22, pp. 1185-1186, 1986.

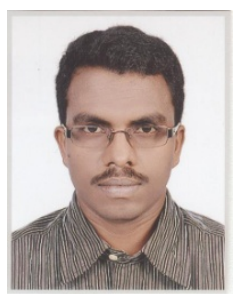

S. K. Das is an Assistant Professor at the Department of Electronics and Communication engineering, National Institute of Technology, Rourkela India. He received his MS from IISc Bangalore, India, in 2002. He has worked in many organizations both in India as well as in abroad in various capacities. He has served on the Program Committee of a number of international conferences. $\mathrm{He}$ is a member of the IEEE. His research interest includes Computer Networking, Sensor Networking, Optical Networking, Embedded system applications.

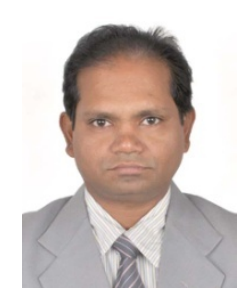

S. K. Patra is a Professor at the Department of Electronics and Communication engineering, National Institute of Technology, Rourkela, India. He received $\mathrm{PhD}$ Degrees from Edinburg Univ., U.K. in 1998. He has served on the Program Committee of a number of international conferences. $\mathrm{He}$ has more than 50 research publications in international journals and conferences. He is a Senior Member of IEEE, IE, CSI and IETE. His research interests include Signal Processing, Mobile Communication and soft computing. 